PROCEEDINGS OF THE

AMERICAN MATHEMATICAL SOCIETY

Volume 134, Number 5, Pages 1447-1449

S 0002-9939(05)08166-9

Article electronically published on October 13, 2005

\title{
A NOTE ON ANALYTICITY AND FLOQUET ISOSPECTRALITY
}

\author{
ROBERT CARLSON \\ (Communicated by Carmen C. Chicone)
}

\begin{abstract}
A simple argument shows that certain complex Hill's operators have the same Floquet multipliers as the zero potential case. Previous results are extended to include matrix coefficients and some meromorphic potentials.
\end{abstract}

Most of the spectral analysis for Hill's equation

$$
-y^{\prime \prime}+q(x) y=\lambda y, \quad q(x+1)=q(x)
$$

assumes that $q$ is real [3], but there has been some interest in complex potentials. In particular, inverse problems have been studied [4, 7, 8] for

$$
q(x)=\sum_{k=1}^{\infty} q_{k} e^{2 \pi i k x} .
$$

The basis for these analyses is the fact that when $q$ has the form (2), the Floquet multipliers for (1) are the same as for the case $q=0$. A recent paper 9] provides an elementary computational approach to this result.

This note gives a short alternative proof, while also providing some generalizations. It will be convenient to assume that $q(x) \in L_{\text {loc }}^{2}(\mathbb{R})$. Introduce the complex variable $z=x+i t$, and note that (2) implies that $q$ extends analytically to the upper half-plane. This fact was explicitly used in [7, 8].

The first observation is both general and straightforward. Consider the periodic linear homogeneous equation

$$
\sum_{j=0}^{N} A_{j}(z) Y^{(j)}=0, \quad Y(z) \in \mathbb{C}^{N}, \quad A_{j}(z+1)=A_{j}(z), \quad A_{N}(z)=I,
$$

whose $K \times K$ matrix coefficients are analytic in a strip

$$
S=\{z=x+i t \mid a<t<b,-\infty<x<\infty\} .
$$

The Floquet multipliers for (3) are the eigenvalues of the action of translation by the period 1 on the $N K$-dimensional space of solutions.

Let $z_{0}=x_{0}+i t_{0} \in S$. A unique $K \times N K$ matrix solution $\mathcal{Y}\left(z, z_{0}\right)$ of (므), analytic for $z \in S$, is determined by requiring the $N K \times N K$ matrix function

$$
\Psi(z)=\left(\begin{array}{c}
\mathcal{Y}^{(0)} \\
\vdots \\
\mathcal{Y}^{(N-1)}
\end{array}\right)
$$

\footnotetext{
Received by the editors October 18, 2004 and, in revised form, December 16, 2004.
}

2000 Mathematics Subject Classification. Primary 34B30.

(C) 2005 American Mathematical Society Reverts to public domain 28 years from publication 1447 
to satisfy $\Psi\left(z_{0}\right)=I$. The columns of $\mathcal{Y}\left(z, z_{0}\right)$ are a basis of solutions of (3), and the eigenvalues of the Floquet matrix $\Psi\left(z_{0}+1\right)$ are the multipliers. The choice of $z_{0}$ selects a matrix representation of translation by 1 with respect to a particular basis [1, pp. 78-80, 90-91]. This discussion establishes the first lemma.

Lemma 1. The Floquet matrices $\Psi\left(z_{0}+1\right)$ are similar. Their eigenvalues, which are the Floquet multipliers for (3), are independent of $z_{0} \in S$.

By fixing $t=t_{0}$, (3) induces an equation on the real axis, with a basis of solutions given by the columns of $\mathcal{Y}\left(x+i t_{0}, x_{0}+i t_{0}\right)$. The multipliers, inherited from (3), are thus independent of $t_{0}$ (and $x_{0}$ ).

Theorem 2. Suppose $q(x)$ in (1) is a $K \times K$ matrix function $q(x)=q_{0}+$ $\sum_{k=1}^{\infty} q_{k} e^{2 \pi i k x}$ whose $K \times K$ matrix Fourier coefficients satisfy $\sum_{k}\left\|q_{k}\right\|^{2}<\infty$. The Floquet multipliers for (11) are the same as for the case $q=q_{0}$.

Proof. Extend $q$ analytically to $0<t<\infty$. For convenience assume that $x_{0}=0$, and $0 \leq x \leq 1$. Defining $q(x+i \infty)=q_{0}$, the function $t \rightarrow q(\cdot+i t)$, is continuous from $[0, \infty]$ to $L^{2}[0,1]$. For each $\lambda \in \mathbb{C}$ the entries of the Floquet matrix $\Psi(1+i t)$ are continuous functions of $q(\cdot+i t)$. This continuity extends to the trace, and to the other elementary symmetric functions of the eigenvalues which are the coefficients of the characteristic polynomial of $\Psi(1+i t)$. By the previous lemma and continuity these elementary symmetric functions are constant functions of $t \in[0, \infty]$, so agree with the corresponding elementary symmetric functions for (1) when $q=q_{0}$.

Theorem 2 extends to certain meromorphic potentials with trivial monodromy. Suppose that $q(z)$ is a meromorphic function in $\mathbb{C}$. Say that (1) has trivial monodromy if all solutions are single-valued meromorphic functions of $z$ for all $\lambda \in \mathbb{C}$. Examples such as

$$
-y^{\prime \prime}+\frac{2 \pi^{2}}{\cos ^{2}(\pi[z-b])} y=\lambda y
$$

with independent solutions

$$
\begin{gathered}
y_{1}=\sqrt{\lambda} \cos (\sqrt{\lambda}[z-b])+\pi \tan (\pi[z-b]) \sin (\sqrt{\lambda}[z-b]), \\
y_{2}=-\sqrt{\lambda} \sin (\sqrt{\lambda}[z-b])+\pi \tan (\pi[z-b]) \cos (\sqrt{\lambda}[z-b]),
\end{gathered}
$$

may be constructed via Darboux transformations $[2,5,6$. One obtains the following with no important changes to the proof of Theorem 1.

Theorem 3. Suppose that $q(z)$ is meromorphic in $\mathbb{C}$ with trivial monodromy. Assume in addition that $q(z+1)=q(z)$, that $q(z)$ has only finitely many poles in the vertical strip $0 \leq x<1$, and $\lim _{t \rightarrow \infty} q(x+i t)=q_{0}$. Then the Floquet multipliers for (11) are the same as for the case $q=q_{0}$.

\section{REFERENCES}

[1] E. Coddington and N. Levinson, Theory of Ordinary Differential Equations, McGraw-Hill, 1955. MR0069338(16:1022b)

[2] J. Duistermaat and F. Grünbaum, Differential equations in the spectral parameter, Comm. Math. Phys. 103 (1986), 177-240. MR0826863 (88j:58106)

[3] A. Finkel, E. Isaacson and E. Trubowitz, An explicit solution of the inverse periodic problem for Hill's equation, SIAM J. Math. Anal. 18 (1987), no. 1, 46-53. MR0871819 (88d:34037)

[4] M. Gasymov, Spectral analysis of a class of second-order non-self-adjoint differential operators, Functional Analysis and its Applications 14 (1980), 11-15. MR0565091(81c:47048) 
[5] F. Gesztesy and R. Weikard, Picard potentials and Hill's equation on a torus, Acta Math. 176 (1996), 73-107. MR1395670 (97f:14046)

[6] V. Goncharenko and A. Veselov, Monodromy of the matrix Schrödinger equations and Darboux transformations, J. of Phys. A, 31 (1998), 5315-5326. MR1634885 (99g:34016)

[7] V. Guillemin and A. Uribe, Hardy functions and the inverse spectral method, Comm. in Partial Differential Equations, 8 (1983), 1455-1474. MR0714048 (85h:35197)

[8] L. Pastur and V. Tkachenko, Spectral theory of Schrödinger operators with periodic complexvalued potentials, Functional Analysis and its Applications, 22 (1988), 156-158. MR0947620 (89d:34056)

[9] K. Shin, On half-line spectra for a class of non-self-adjoint Hill operators, Math Nachr. 261/262 (2003), 171-175. MR2020394 (2004i:34232)

Department of Mathematics, University of Colorado at Colorado Springs, Colorado Springs, Colorado 80933

E-mail address: carlson@math.uccs.edu 